\title{
Framework for comprehensive enhancement of brain tumor images with single-window operation
}

\author{
Deepthi Murthy T. S. ${ }^{1}$, Sadashivappa G. ${ }^{2}$ \\ ${ }^{1}$ School of Electronics and Communication Engineering (ECE), REVA University, India \\ ${ }^{2}$ Department of Telecommunication Engineering, RV College of Engineering, India
}

\begin{tabular}{l} 
Article Info \\
\hline Article history: \\
Received Aug 10, 2018 \\
Revised Sep 13, 2019 \\
Accepted Sep 27, 2019 \\
\hline
\end{tabular}

Keywords:

Image enhancement

Medical image

Optimization

Preprocessing

Visual quality

\begin{abstract}
Usage of grayscale format of radiological images is proportionately more as compared to that of colored one. This format of medical image suffers from all the possibility of improper clinical inference which will lead to error-prone analysis in further usage of such images in disease detection or classification. Therefore, we present a framework that offers single-window operation with a set of image enhancing algorithm meant for further optimizing the visuality of medical images. The framework performs preliminary pre-processing operation followed by implication of linear and non-linear filter and multi-level image enhancement processes. The significant contribution of this study is that it offers a comprehensive mechanism to implement the various enhancement schemes in highly discrete way that offers potential flexibility to physical in order to draw clinical conclusion about the disease being monitored. The proposed system takes the case study of brain tumor to implement to testify the framework.
\end{abstract}

Copyright $(2020$ Institute of Advanced Engineering and Science. All rights reserved.

\section{Corresponding Author:}

Deepthi Murthy T S,

School of Electronics and Communication Engineering (ECE),

REVA University,

Bangalore, India.

Email: deepthiresearch1983@gmail.com

\section{INTRODUCTION}

There has been a tremendous revolution in the area of medical image processing that is essentially meant for accurately diagnosing the disease [1]. The existing techniques of medical imaging include ultrasound, Magnetic Resonance Imaging (MRI), Positron Emission Tomography (PET), CT (Computed Tomography) etc [2]. The degree of improvement in such advance imaging so high that even a microstructure and tissue can be seen during the visual analysis [3]. The existing MRI offers multiple slices of the scanned region to have better look into the suspected region of human body [4]. Most importantly, there are various advance scientific research work e.g. [5, 6], which claims that medical imaging could be possibly used for automated diagnosis as well as making the automatic inference of the diagnosed disease using sophisticated classification techniques. Improvement in the classification techniques potentially assists in detecting the stage of the disease with accurate clinical information [7].

However, such research ideas are only limited to research papers and yet not make out to the commercial markets. There are multiple reasons behind this. It has been seen that majority of the research approaches towards disease diagnosis and classification uses datasets and very less on real-time images. It is because data set images are already pre-processed and free from any form of errors so that it becomes easy to check the applicability of research-based algorithms on such dataset images. However, in real-time medical images do suffer from some realistic problems that are less addressed in existing research work although some research papers have highlighted few facts about it [8]. The first issues is that if the pre-processing of the medical images are not carried out effectively than it will lead the other algorithm to 
extract error-prone information that will lead to wrong direction of research work. Second issues in medical image processing are related to the grayscale form of the image where it is quite difficult to make out the actual location of suspected disease. Inspite of colored radiological images being used in special cases, usage of grayscale version of medical image is frequently utilized in real-time environment. At present, there are various forms of pre-processing algorithms [9] as well as denoising algorithm [10], but very few of them have been tested with complicated cases of medical images.

All the existing pre-processing techniques focuses on implementing some common methods e.g. wavelets, Retinex, contrast enhancement, filters, histogram, etc. All these techniques are good enough for certain set of images and don't offer similar scale of performance if the implementation environments as well as the datasets are altered. This will eventually mean that existing techniques doesn't necessarily ensure uniformity of the enhancement performance. It is quite likely that a physician might want to check one radiological image from multiple angles within a shortest span of time. At present, apart from simple form of contrast, brightness, sharpness, and opacity, there are not much form of enhancement that a physician can do in order to make decision of clinical inference. This is a major research gap which calls for investigating some reliable techniques for solving such issues. Therefore, we present a very simple and novel framework where medical image can be subjected to multiple form of enhancement using set of algorithms that are not only computational friendly but also offer faster response time.

This paper presents discussion of one such algorithm where series of image enhancement techniques have been implemented. Section 1 discusses about the existing literatures where different medical image enhancing techniques are discussed followed by discussion of research problems explored from the existing research approaches in Section 1.1 and proposed solution to solve such research problem in 1.2. Section 2 discusses about image enhancing algorithm implementation that is meant for offering comprehensive image enhancing operation in highly cost effective way followed by discussion of result analysis in Section 3. Finally, the conclusive remarks are provided in Section 4.

This section discusses about the existing research approaches towards the medical image enhancement. The most recent work carried out by Yelmanova and Romanyshyn [11] have focused on involuntary contrast enhancement using histogram. Zhao and Zhou [12] have presented a masking principle for the purpose embedding the multiplicative steps in it that significantly enhances the medical images. The adoption of Verilog scheme was seen in the work of Chiuchisan [13] for prototyping the image enhancing scheme. Usage of sliding decomposition approach for analyzing the detailed feature of medical images in the work of Liang and Si [14]. Gong et al. [15] have introduced chaotic approach in order to enhance the performance of immune algorithm for enhancing the brain images. Li and Kang [16] have implemented wavelet based approach in order to enhance the resolution of medical image using both conventional wavelets as well as inverse wavelets. Soloperto et al. [17] have introduced a clinical approach using Halloysite nanotubes for investigating its trends during the enhancement of medical images.

Xue [18] have used contrast enhanced index for improving the medical images using Shearlet-based approach. Usage of contourlet transform was seen in the work of Zhou et al. [19] along with a Laplace pyramid for an effective control on multiple coefficients. Chaira [20] used a fuzzy logic for addressing similar problems. Dhinagar and Celenk [21] have used histogram as well as homomorphic filtering for enhancing the image generated from ultrasound. The work carried out by Hua et al. [22] have used YoungHelmholtz based transformation scheme in order to enhance colored radiological images. Kurt et al. [23] have used adaptive histogram as well as anisotrophic filters in order to perform enhancement of medical images. Xiurong [24] have implemented a masking algorithm of adaptive nature in order to effectively control the sharpening of the medical images.

Fan et al. [25] have constructed an enhanced look up table for improving the contrast in multi-scale factor. Adoption of context and gradient smoothness towards enhancing the medical image was seen in the work of Jing and Jie [26]. Literatures have also witnessed usage of non-linear techniques for enhancing medical images as seen the work carried out by Hossain et al. [27]. The authors have also used histogram matching as well as logarithmic transform scheme to further enhance it. Jiang et al. [28] have used contourlet transform using invariance approach in order to assists in image representation and mitigate noise level in medical images. Usage of wavelet transform was observed in the implementation carried out by Yang et al. [29] for performing medical image enhancement. Therefore, it can be seen that there are different techniques available in literatures with an emphasis on addressing image enhancement problems, however, majority of the existing schemes have nearly similar forms of implementation schemes with lesser availability of journals addressing such problems.

However, such schemes are quite capable of addressing particular problems and would succumb to a failure if the test environment is changed. Such identified issues are outlined in next section in the form of research problem. Bangare et al. [30] have illustarted to find out tumor ripped lesions to be make better using fluorescence image resolution. Kahina et al. [31] have demonstarted Viterabi decoder with the window 
technique. Verma and Sharma [32] have presented an optimal image improvement technique for color images by protecting their strength.

The research problems identified from literature review are as follows:

a. Existing system are too much specific about the enhancement and therefore they cannot caterup the processing requirements of multiple forms of radiological images.

b. Single algorithm or set of algorithm addressing multiple forms of enhancement issues of medical images are very few to explore in existing system.

c. Usage of existing schemes doesn't offer a single-handed function where there is a scope of further enhancement or rather optimizing the enhancement process.

d. Existing approaches are also mathematically complex in implementation when it comes for computational process leading to longer response time with external dependencies.

Therefore, the problem statement of the proposed study can be stated as "Designing a single set of algorithm with one-window operation that assists in multiple-level of enhancement as per the clinical investigation with retention of cost effectiveness in approach is quite challenging.

The proposed system is an extension of our prior work [33] where we have introduced segmentation of brain tumor. This work will be considered as complimentary for the segmentation process as it offers furnishing more pixel-based enhancement on the brain tumor images. The Figure 1 shows the schematic diagram of the proposed system is as follows:

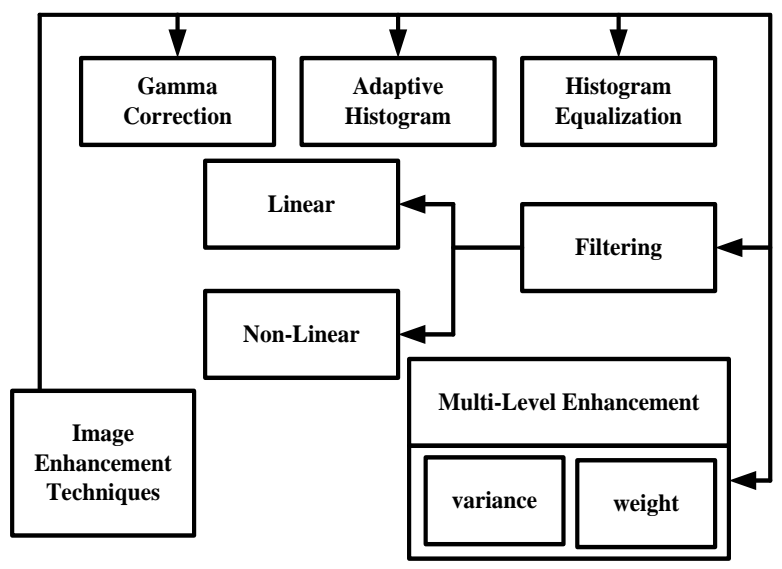

Figure 1. Schema of proposed methodology

The proposed technique offers comprehensive individual methods in one window operation that can perform enhancement of the given radiological image. The proposed system consists of three core modules i.e. preliminary preprocessing, applying filters, and multi-level enhancement. The preliminary preprocessing operation consists of applying gamma correction, adaptive histogram, and histogram equalization. The second process is about applying filter of two forms i.e. linear and non-linear forms in order to mitigate all forms of distortions in the frequeny levels. The third process is about multiple forms of enhancement where the emphasis is given to formulate an empirical expression in order to investigate the impact of statistical feature with the enhancement process. The proposed system uses variance and weight in multiple orders for investigating the impact of it towards the enhancement of an image.

The overall outcome was assessed using Peak Signal-to-Noise Ratio (PSNR). The proposed scheme offers the physical with various alternatives of image enhancement as there is no particular enhancement technique that can have equal performance on different forms of radiological images in existing system. Hence, the proposed system offers a single window operation, where one radiological image of brain tumor can be investigated more easily owing to availability of different forms of image enhancement technique. The next section discusses about the algorithm implementation.

\section{ALGORITHM IMPLEMENTATION}

This algorithm is responsible for performing multiple forms of enhancement of brain tumor images in order to assist the diagnosis process for better visualization. Hence, this algorithm implements a series of image enhancement techniques that offers better decision process during closer investigation of radiological 
images of brain tumor. The first part of the study implies gamma correction method. The gamma correction method is mathematically represented as,

$$
G(x, y)=[f(x, y)]^{\gamma}
$$

Where, $G(x, y) \rightarrow$ intensity of the output image, $\mathrm{f}(\mathrm{x}, \mathrm{y}) \rightarrow$ intensity values of the input image, $\mathrm{T} \rightarrow$ transformation function, $\mathrm{x}, \mathrm{y} \rightarrow$ coordinates of the image pixels, $\gamma \rightarrow$ the constant value defined as gamma value. The second process deals with histogram equalization. Considering the Rayleigh distribution in AHE, A histogram of bell shaped curve is produced, which can be defined as a function given as

$$
g=g_{\min }+\left[2 \times \alpha^{2} \ln \frac{1}{1-P(f)}\right]^{0.5}
$$

Where, $g \rightarrow$ distribution function, (in this case it is Rayleigh distribution), $g_{\min } \rightarrow$ Minimum pixel value, and $\mathrm{P}(\mathrm{f}) \rightarrow$ cumulative probability distribution function which is a non-negative real scalar representing a distribution parameter. The mathematical expression for applying linear filtering is as below:

$$
I_{p}(i, j)=\sqrt{[\exp (f(i, j))]^{2}}
$$

Where, $I_{p} \rightarrow$ enhanced image. In non-linear filtering, the image is defined by,

$$
I=f(i, j)
$$

Where, $\mathrm{i} \rightarrow$ number of rows and $\mathrm{j} \rightarrow$ number of columns, and $\mathrm{L} \rightarrow$ logarithmic single channel intensity image to be processed, niter $\rightarrow$ number of iterations, Enc_img $\rightarrow$ raw enhanced output image. To obtain the maximum colour variance of the channel, Maximum $=\max (\mathrm{L})$

$$
O P=\text { Maximum } \times \sum_{i, j}^{N} a_{i j}
$$

Where, $a \rightarrow 1$. To perform initial shift,

$$
\text { shift }=\sum_{i, j=1}^{N-1} 2^{\left(\log _{2}(\min (i, j))\right)-1}
$$

Initializing old product, the horizontal and vertical step is given by

$$
\begin{aligned}
& I_{p}=\left\{\begin{array}{l}
I_{p 1} \mid i+j>0 \\
I_{p 2} \mid i+j<0
\end{array}\right. \\
& I_{p 1}=\left(\sum_{1}^{N-i} \sum_{1}^{N-j} O P(i, j)\right)+\left(\sum_{i+1}^{N} \sum_{j+1}^{N} R R(i, j)\right)-\left(\sum_{1}^{N-i} \sum_{1}^{N-j} R R(i, j)\right) \\
& I_{p 2}=\left(\sum_{1-i}^{N} \sum_{1-j}^{N} O P(i, j)\right)+\left(\sum_{1}^{N+i} \sum_{1}^{N+j} R R(i, j)\right)-\left(\sum_{1-i}^{N} \sum_{1-j}^{N} R R(i, j)\right) \\
& \text { shift }=\frac{\text { shift }}{2}, \\
& N P=\frac{(I P+O P)}{2} \\
& O P=N P, \text { where, } N P \rightarrow \text { enhanced image }
\end{aligned}
$$

In Multilevel Enhancement, the Initial parameters are a $\rightarrow$ weight initialized to a vector (i.e. $a_{1}, a_{2}, a_{3}, a_{4}, a_{5}$ ), var $\rightarrow$ variance initialized to a vector of finite values (i.e. $w_{1}, w_{2}, w_{3}, w_{4}, w_{5}$ ). Enhancement with respect to primary image enhancement is given as,

$$
G(i, j)=\sum_{i, j=1}^{N-1, M-1} k 1 \cdot \exp \left(-\frac{\left(i^{2}+j^{2}\right)}{v a r^{2}}\right)
$$

Where, $\mathrm{G} \rightarrow$ image with respect to Gaussian distribution function, I $\rightarrow$ original image, $\mathrm{x}$, $\mathrm{y} \rightarrow$ pixel coordinates. Performing convolution for the (1). 


$$
\operatorname{conv}=I \otimes G
$$

Where, conv $\rightarrow$ convoluted image, computing the difference and applying log to both the variables, we get

$$
I_{p}=\log (I+1)-\log (\text { conv })
$$

Where, $I_{p} \rightarrow$ enhanced image

The significant steps of the algorithm are as follows:

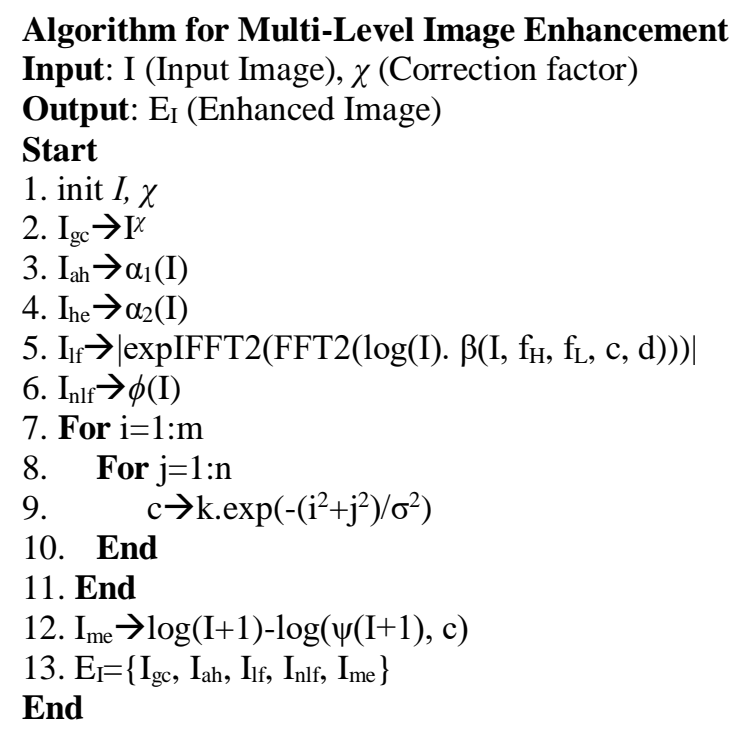

The algorithm first performs gamma correction in order to encoding as well as decoding the luminance factor for a given value of correction factor $\chi$ (Line-2) in order to obtain gamma corrected image $I_{g c}$. The algorithm also allows implementing adaptive histogram $\alpha_{1}$ and histogram equalization $\alpha_{2}$ in order to obtain enhanced image $\mathrm{I}_{\mathrm{ah}}$ (Line-3) and $\mathrm{I}_{\mathrm{he}}($ Line-4). The next part of the algorithm implements linear filtering process using multiple forms of input parameters e.g. $f_{H}$ (high-frequency), $f_{L}$ (low-frequency), c (constant that controls the slope peaks), d (threshold frequency). This process is also associated with applying Fourier and inverse Fourier transform in order to obtain an enhanced image $\mathrm{I}_{\mathrm{lf}}$ (Line-5). The next part of the algorithm focuses on implementing non-linear filter $\phi$ (Line-6).

The input to the function $\phi$ is a logarithmic single-channel intensity image that is required to be enhanced as well as iterations too whereas the output of this process is enhanced image accomplished from non-linear filtering. This step of the algorithm uses a shift operator that initiates with initializing the maximum color value in the image followed by design of initial shift as wel as old product towards horizontal and vertical steps. Finally, the shift operators are updated. The next part of the algorithm introduces multiple-level of enhancement where the input image has the scope to get itself enhance depending on the numerical inputs offered by the user. For this purpose, the algorithm takes weight as well as variance. Than for all the values of rows (Line-7) and column (Line-8), the algorithm implements an empirical expression of $c$ (Line-9) that depends on image dimensions ( $i$-row and $j$-column) and variance $\sigma$ (Line-9). This step is followed by computation of finally enhanced image $I_{m e}$ by applying logarithmic function and convolution operation on the image (Line-12).

The algorithm offers three different forms of enhancement in the multiple levels. The primary level of enhance is carried out by only one values of weight and variance, whereas the secondary image enhancement level is carried out by using dual values of weight and variance. Similarly, the teriary image enhancement is carried out using three values of weight and variance. Incorporation of multiple forms of weight and variable offers flexibility to assess different forms of radiological images. One interesting fact about the proposed system is that it offers multiple forms of simple image enhancement techniques in single window operation that offers the significant level of advantage for the physician to diagnose the different forms of brain tumor images with more customized forms of enhancement. At the same time, majority of the filtering process is carried out with an equal emphasis on linear and non-linear filtering techniques, which is one of the great methods for addressing the multiplicative noise (as radiological images do have multiplicative noises). Moreover, as the radiological images of brain tumor are normally grayscale therefore, this algorithm offers a set of enhancement techniques (Line-13) that optimizes the appearance of 
the grayscale images with reduced iteration and highly in progressive forms. Therefore, the proposed system can be claimed to offer a cost effective solution to enhance the brain tumor images within inclusion of any complex and iterative parameters in the filter design.

\section{RESULT ANALYSIS}

This section discusses about the outcomes obtained from implementing the proposed system. The analysis of image enhancement was carried out in brain dataset where certain samples of visual outcomes were exhibited in Figure 2 where Figure 2(a) represents original sample image, Figure 2(b) represents gamma corrected image, Figure 2(c) represents image acquired from adative histogram, Figure 2(d) represents image obtained from histogram equalization. The outcome of linear and non-linear filtering can be seen in Figure 2(e) and Figure 2(f). The multiple forms of enhancement for primary, secondary, and tertiary forms with multiple weights and variance can be seen in Figure 2(g), Figure 2(h), and Figure 2(i) respectively. Finally, Figure 2(j) shows outcome of joint forms of multiple enhancement by maximizing the number of weights and variance level.
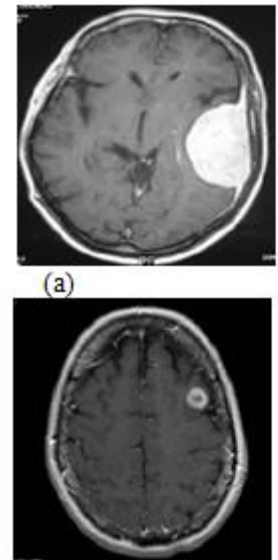

(f)
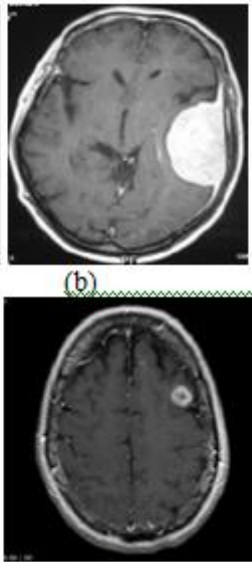

(g)
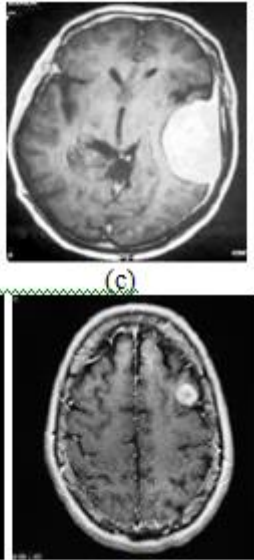

(h)

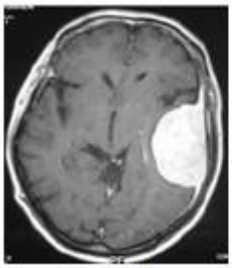

(d)

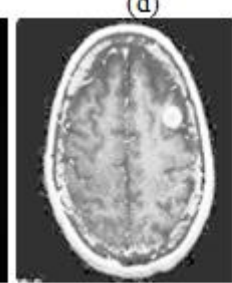

(i)
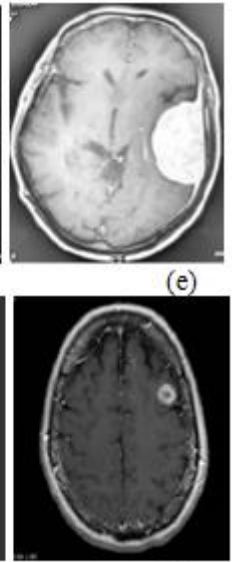

(j)

Figure 2. Visual outcomes of proposed system

We also closely monitor the outcome of the PSNR for all the implemented image enhancement techniques and found that each technique have different forms of pattern based on its visual quality. However, with the outcome shown in Figure 3, there should be any conclusion drawn about effectiveness of any specific technique as we have perform this analysis by uniformly distributing multiple weight and variance values. Looking into this outcome, it will not mean that multi-level enhancement offers lesser PSNR is ineffective while gamma correction is highly effective. As for different forms of images, the outcomes differ to some extent depending upon the visual complexity of an image.

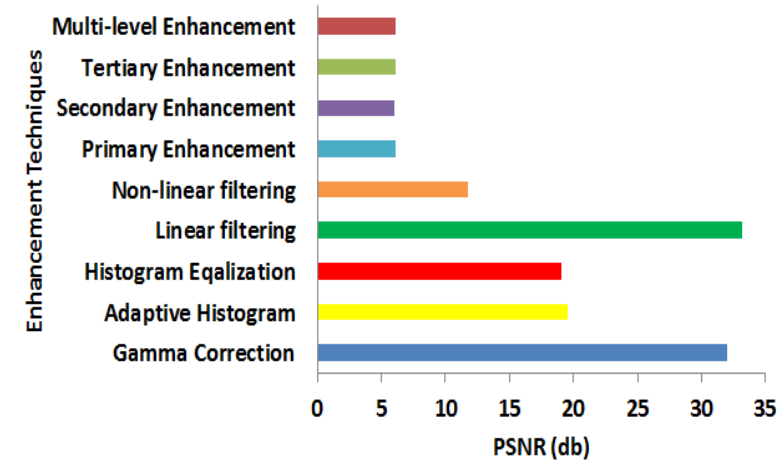

Figure 3. Comparative performances of image enhancement techniques 
It can only be said that proposed system offers a tool that allows the physician to perform thorough investigation of the radiological image of a brain tumor so that the diagnosis is carried out in proper manner. At the same time, the algorithm takes minimum of 0.052755 seconds and maximum of 1.223537 seconds in order to provide the response of enhanced image of brain tumor. The response time is quite faster and there is accumulation of any memory or dependency of any heuristics in order to perform the analysis. Hence, it can be said that proposed system offers wide ranges of image enhancement techniques in one window operation with faster response to enhanced image.

\section{CONCLUSION}

From the discussion presented in this paper, it is very much clear that it is feasible to construct a framework using sub-set of algorithms for offering wide-range of enhanced image. The first contribution of this paper is its cost effectiveness as in order to implement this scheme in reality there is no much dependency of any external computational or hardware-based parameter and neither there is any external dependencies. The second contribution of this paper is that it offers a physician to view the given enhancement outcome in their possible choices which offers them better scope of decision making. The third contribution of this approach is that statistical parameters e.g. weight and variance plays a significant role that can offer more comprehensive extraction of information to offer more granularities in the enhanced images for diagnosis purpose. Our future work will be completely in the direction of making classification of diseases on the top of this framework.

\section{REFERENCES}

[1] W. Birkfellner, Applied Medical Image Processing, Second Edition: A Basic Course, Taylor \& Francis, 2016.

[2] D. V. Sahani and A. E. Samir, Abdominal Imaging, E-Book: Expert Radiology Series, Elsevier Health Sciences, 2016.

[3] K. W. Lai, et al., Advances in Medical Diagnostic Technology, Springer Science \& Business Media, 2014.

[4] C. H. Chen, Computer Vision in Medical Imaging, World Scientific, 2014.

[5] A. Das, et al., "Detection of abnormal regions of precancerous lesions in digitised uterine Cervix images," 2014 International Electrical Engineering Congress (iEECON), Chonburi, pp. 1-4, 2014.

[6] S. Khobragade, et al., "Automatic detection of major lung diseases using Chest Radiographs and classification by feed-forward artificial neural network," 2016 IEEE 1st International Conference on Power Electronics, Intelligent Control and Energy Systems (ICPEICES), Delhi, pp. 1-5, 2016.

[7] R. Chandra, et al., "On the Opportunities and Challenges in Microwave Medical Sensing and Imaging," IEEE Transactions on Biomedical Engineering, vol. 62, pp. 1667-1682, 2015.

[8] F. L. Lizzi and E. J. Feleppa, "Image Processing and Pre-Processing for Medical Ultrasound," ACM_roceedings of the 29th Applied Imagery Pattern Recognition Workshop, pp. 187, 2000.

[9] B. Chitradevi and P. Srimathi, “An Overview on Image Processing Techniques," International Journal of Innovative Research in Computer and Communication Engineering, 2014.

[10] S. Preethi and D. Narmadha, "A Survey on Image Denoising Techniques," International Journal of Computer Applications, vol. 58, pp. 27-30, 2012.

[11] E. S. Yelmanova and Y. M. Romanyshyn, "Medical image contrast enhancement based on histogram," 2017 IEEE 37th International Conference on Electronics and Nanotechnology (ELNANO), Kiev, pp. 273-2, 2017.

[12] Z. Zhao and Y. Zhou, "PLIP based unsharp masking for medical image enhancement," 2016 IEEE International Conference on Acoustics, Speech and Signal Processing (ICASSP), Shanghai, pp. 1238-1242, 2016.

[13] I. Chiuchisan, "An approach to the Verilog-based system for medical image enhancement," 2015 E-Health and Bioengineering Conference (EHB), Iasi, pp. 1-4, 2015.

[14] L. Liang and Y. Si, "Medical image enhancement using sliding weighted empirical mode decomposition," 2015 IEEE International Conference on Information and Automation, Lijiang, pp. 3145-3148, 2015.

[15] T. Gong, et al., "Improved immune algorithm for medical image enhancement," 2015 International Workshop on Artificial Immune Systems (AIS), Taormina, pp. 1-7, 2015.

[16] X. W. Li and Y. X. Kang, "A novel medical image enhancement method based on wavelet multi-resolution analysis," 2015 8th International Conference on Biomedical Engineering and Informatics (BMEI), Shenyang, pp. 727-731, 2015.

[17] G. Soloperto, et al., "Multiparametric Evaluation of the Acoustic Behavior of Halloysite Nanotubes for Medical Echographic Image Enhancement," IEEE Transactions on Instrumentation and Measurement, vol. 63, pp. 1423-1430, 2014.

[18] Q. Xue, "Enhancement of medical images in the shearlet domain," Proceedings of 2013 3rd International Conference on Computer Science and Network Technology, Dalian, pp. 235-238, 2013.

[19] F. Zhou, et al., "Medical image enhancement based on NSCT," IET International Conference on Smart and Sustainable City 2013 (ICSSC 2013), Shanghai, pp. 166-169, 2013.

[20] T. Chaira, "Medical image enhancement using intuitionistic fuzzy set," 20121 st International Conference on Recent Advances in Information Technology (RAIT), Dhanbad, pp. 54-57, 2012. 
[21] N. J. Dhinagar and M. Celenk, "Ultrasound medical image enhancement and segmentation using adaptive homomorphic filtering and histogram thresholding," 2012 IEEE-EMBS Conference on Biomedical Engineering and Sciences, Langkawi, pp. 349-353, 2012.

[22] L. Hua, et al., "A new color medical image enhancement method," IET International Conference on Information Science and Control Engineering 2012 (ICISCE 2012), Shenzhen, pp. 1-5, 2012.

[23] B. Kurt, et al., "Medical images enhancement by using anisotropic filter and CLAHE," 2012 International Symposium on Innovations in Intelligent Systems and Applications, Trabzon, pp. 1-4, 2012.

[24] T. Xiurong, "The application of adaptive unsharp mask algorithm in medical image enhancement," Proceedings of 2011 Cross Strait Quad-Regional Radio Science and Wireless Technology Conference, Harbin, pp. 1368-1370, 2011.

[25] B. Fan, et al., "Medical image enhancement based on modified lut-mapping derivative and multi-scale layer contrast modification," 2011 4th International Congress on Image and Signal Processing, Shanghai, pp. 696-703, 2011.

[26] L. Jing and X. Jie, "Research and Implementation on the Digital Medical Image Enhancement Methods Based on Gradient Smooth and Context Smooth," 2011 Seventh International Conference on Computational Intelligence and Security, Hainan, pp. 1193-1196, 2011.

[27] M. F. Hossain, et al., "Medical image enhancement based on nonlinear technique and logarithmic transform coefficient histogram matching," IEEE/ICME International Conference on Complex Medical Engineering, Gold Coast, QLD, pp. 58-62, 2010.

[28] A. Jiang, et al., "Medical Image Enhancement Algorithm Based on Contourlet Transform," 2010 First International Conference on Pervasive Computing, Signal Processing and Applications, Harbin, pp. 624-627, 2010.

[29] Y. Yang, et al., "Medical image enhancement algorithm based on wavelet transform," Electronics Letters, vol. 46, pp. 120-121, 2010.

[30] S. L. Bangare, et al., "Neuroendoscopy Adapter Module Development for Better Brain Tumor Image Visualization," International Journal of Electrical and Computer Engineering (IJECE), vol. 7, no. 6, pp. 3643-3654, 2017.

[31] R. Kahina and A. Bassou, "Improving The Performance of Viterbi Decoder using Window System," International Journal of Electrical and Computer Engineering (IJECE), vol. 8, no. 1, pp. 611-621, 2018.

[32] Verma, et al., "Intensity Preserving Cast Removal in Color Images Using Particle Swarm Optimization," International Journal of Electrical \& Computer Engineering (IJECE), vol. 7, no. 5.pp. 2581-2595, 2017.

[33] T. S. D. Murthy and G. Sadashivappa, "Brain tumor segmentation using thresholding, morphological operations and extraction of features of tumor," IEEE-International Conference on Advances in Electronics Computers and Communications, Bangalore, pp. 1-6, 2014.

\section{BIOGRAPHIES OF AUTHORS}

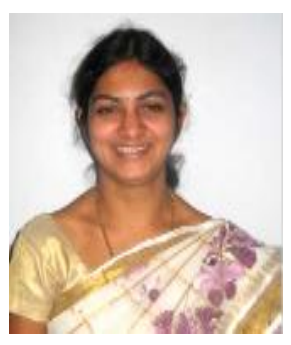

Deepthi Murthy T.S, received the BE (Electronics and Communication) Degree from VTU, India in 2005, and the M.Tech (Digital Electronics and Communication) degree from VTU, India in 2007. She has 10 years of teaching experience. She is currently working as an Assistant Professor in REVA University, Bangalore; her research interests include Medical Imaging, Signal Processing and Applications.

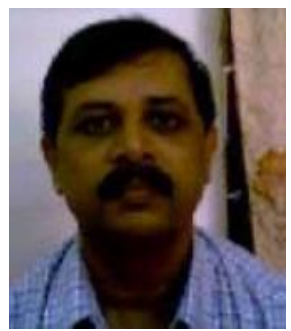

Dr. G. Sadashivappa, has obtained his doctoral degree from VTU, Belgaum. He has 32 years of teaching experience and 10 years of research experience. He has authored two text books. He has chaired many international conferences. He was the Principle Investigator for the project "On Study and development of Image Compression Algorithms for Satellite Images" supported by ISRO. He is currently professor at Telecommunication Engineering Department, RVCE, Bangalore. His research area of interest is Fiber Optic communication, Medical Imaging, Signal Processing \& applications. He has published many international journals. 